\title{
ARANEOFAUNA (ARACHNIDA: ARANEAE) DE LA SABANA DE PAJÓN EN EL PARQUE NACIONAL VALLE NUEVO, REPÚBLICA DOMINICANA Y SU COMPARACIÓN ENTRE DOS TEMPORADAS DEL AÑO*
}

\author{
Solanlly Carrero Jiménez y Gabriel de los Santos
}

Museo Nacional de Historia Natural "Prof. Eugenio de Jesús Marcano". Calle César Nicolás Penson, Plaza de la Cultura Juan Pablo Duarte, 10204, Santo Domingo, República Dominicana. s.carrero@mnhn.gov.do; g.delossantos@mnhn.gov.do

\section{RESUMEN}

Se registran y comparan la riqueza, diversidad y abundancia de las arañas asociadas a la sabana de pajón dentro del Parque Nacional Valle Nuevo entre una temporada lluviosa y una seca. Se establecieron cuatro parcelas de $20 \times 20 \mathrm{~m}$ (dos en cada temporada) y en cada una se delimitaron cinco cuadrículas de $2 \times 2 \mathrm{~m}$. En total se colectaron 222 especímenes pertenecientes a 19 familias, 23 géneros y 35 especies. No hubo diferencias significativas entre las temporadas en los índices de Shannon $(P=0.86)$, Simpson $(P>0.99)$ y Pielou $(P=0.41)$. El índice de Similitud de Jaccard entre las temporadas fue de 0.46 . La mayor cantidad de especies estimadas fue de 46 (Chao 2) para la temporada seca y $39(A C E)$ para la lluviosa. En la temporada lluviosa, la familia Salticidae fue la más abundante (21.54\%) y Linyphiidae la más diversa (cuatro especies); la especie más abundante fue Barronopsis barrowsi (Gerstch), 18.96\%. En la temporada seca, la familia Anyphaenidae presentó los más altos valores de abundancia y riqueza específica (44.31\%; cuatro especies), siendo Anyphaena pusilla Bryant la especie más abundante (21.69\%).

Palabras clave: diversidad, abundancia, arañas, sabana de pajón, Valle Nuevo, La Hispaniola.

Title: Araneofauna (Arachnida: Araneae) of the highland savanna in Valle Nuevo National Park, Dominican Republic and its comparison between two seasons of the year.

\section{ABSTRACT}

Richness, diversity and abundance of spider species associated with the highland savanna in Valle Nuevo National Park is recorded and compared between a wet and a dry season. Four plots of $20 \times 20 \mathrm{~m}$ (two in each season) were established and five grids of $2 \times 2 \mathrm{~m}$ were delimited within each plot. A total of 222 specimens belonging to 19 families, 23 genera and 35 species were collected. No significant difference was found between seasons for the Shannon index $(P=0.86)$, Simpson index $(P>0.99)$ and Pielou index $(P=0.41)$. The Jaccard similarity index between seasons was 0.46 . The highest quantity of estimated species was 46 (Chao 2) for the dry season and $39(A C E)$ for the rainy season. In the rainy season, Salticidae was the most abundant family (21.54\%) and Linyphiidae the most diverse (four species); Barronopsis barrowsi (Gerstch) was the most abundant species (18.96\%). In the dry season, the family Anyphaenidae was the most diverse and abundant (four species and $44.31 \%$ ) and Anyphaena pusilla Bryant the most abundant species $(21.69 \%)$.

Keywords: diversity, abundance, spiders, highland savanna, Valle Nuevo, Hispaniola.

\footnotetext{
* Este trabajo forma parte de los resultados del estudio "Ecología de la fauna asociada al suelo en la sabana de pajón del Parque Nacional Juan Bautista Pérez Rancier (Valle Nuevo) y su respuesta a los impactos de la agricultura y el fuego" del Museo Nacional de Historia Natural "Profesor Eugenio de Jesús Marcano", financiado por el Fondo Nacional de Innovación y Desarrollo Científico y Tecnológico (FONDOCyT) del Ministerio de Educación Superior, Ciencia y Tecnología (MESCyT).
} 


\section{INTRODUCCIÓN}

Con más de 44,500 especies descritas (Platnick, 2014) las arañas conforman uno de los grupos megadiversos del planeta y se les reconoce como un componente importante de los ecosistemas donde habitan, especialmente en las cadenas tróficas de los invertebrados (Coddington y Levi, 1991).

De acuerdo con Mineo et al. (2010), se conoce poco sobre la influencia de la estacionalidad en la fauna de arañas de las regiones de sabanas, este autor cita los trabajos de Russell-Smith (2002), Whitmore et al. (2002) y Modiba et al. (2005) entre los mejores documentados. El factor estacionalidad sí ha sido considerado en estudios sobre otros ecosistemas (e.g. Lubin, 1978; Churchill, 1998; Arango et al., 2000; Sudhikumar et al., 2005; Deza y Andía, 2009). En la República Dominicana, sin embargo, los estudios ecológicos sobre arañas han sido tan escasos que solo se pueden citar los trabajos realizados por Sánchez-Ruiz et al. (2009) y Carrero Jiménez (2014); en lo que respecta al Parque Nacional Valle Nuevo, la casi generalidad de los estudios se han enfocado en aspectos taxonómicos (Bryant, 1943, 1945, 1948; Platnick y Shadab, 1974; Alayón, 2011).

\section{OBJETIVOS}

- Caracterizar y comparar la araneofauna de la sabana de pajón del Parque Nacional Valle Nuevo durante una temporada lluviosa y otra seca.

\section{MATERIALES Y MÉTODOS}

Área de estudio. Los muestreos fueron realizados en las localidades La Pirámide y La Lechuguilla, provincia La Vega; ambas dentro del Parque Nacional Valle Nuevo, localizado en el sureste de la Cordillera Central y ubicado entre en los paralelos 18 $36^{\prime} 10^{\prime \prime}$ - 18 $8^{\circ} 57^{\prime} 52^{\prime \prime}$ latitud norte y $70^{\circ} 26^{\prime} 56^{\prime \prime}-70^{\circ} 51^{\prime} 44^{\prime \prime}$ longitud oeste (Ceballos, 2006). Este parque ocupa un área aproximada de $910 \mathrm{~km}^{2}$ y posee una cobertura boscosa de unos $390 \mathrm{~km}^{2}$, dentro de los cuales las sabanas de pajón (donde predomina la gramínea endémica Danthonia domingensis Hackel et Pilger, 1909) se localizan entre los pinares del altiplano, en la porción central del parque. Presenta una precipitación media anual de 1,026.4 mm, con máximos de lluvias en mayo y una temperatura promedio anual de $18.2^{\circ} \mathrm{C}$ (Núñez et al., 2006).

Muestreo. Se establecieron cuatro parcelas de 20x20 m dentro de la sabana de pajón, dos en La Pirámide y dos en La Lechuguilla (trabajándose dos por temporada). En cada parcela se delimitaron cinco cuadrículas de $2 \times 2$ m: una en cada esquina y otra en el centro (Sánchez-Ruiz et al., 2009, con modificaciones).

Temporada lluviosa (TLL): a) Parcela 1 (PLL1); localidad La Pirámide (12/VI/2010); coordenadas, UTM 19Q 331313m.E 2069156m.N; altitud 2,395 m; temperatura del suelo al momento del muestreo, $13.8 \mathrm{C}^{\circ}$. La asociación vegetal y su cobertura según Peguero (2010), consistió en un herbazal con algunos arbustos y árboles dispersos, principalmente de Baccharis myrsinites y Pinus occidentalis. La cobertura vegetal fue aproximadamente $85 \%$. El estrato herbáceo, con altura de $0.5 \mathrm{~m}$, estuvo dominado por $D$. domingensis; además, formando masas significativas en el suelo se encontraron: Cladonia cf. alpestris y Stereocaulon myriocarpum. b) Parcela 2 (PLL2); localidad La Lechuguilla (26/VIII/2010); UTM 19Q 331501m.E 2070693m.N; altitud 2,412 m; temperatura del suelo al momento del muestreo, $13.5 \mathrm{C}^{\circ}$. Herbazal con predominio de $D$. domingensis, formando una masa bien compacta, con altura de hasta $0.6 \mathrm{~m} \mathrm{y}$ una cobertura vegetal entre el 85 y $90 \%$. 
Temporada seca (TS): a) Parcela 1 (PS1); localidad La Lechuguilla (16/I/2011); UTM 19Q 331530m.E 2070703m.N; altitud, 2,362 m; temperatura del suelo al momento del muestreo, $8 \mathrm{C}^{\circ}$. La asociación vegetal y su cobertura sigue a Peguero (2010). La composición florística fue prácticamente la misma de PLL2, agregándose sólo un individuo de $P$. occidentalis. Cobertura aproximadamente de $75-80 \%$, la especie dominante fue $D$. domingensis; se encontraron con relativa abundancia, los líquenes Cladonia cf. alpestris y $S$. myriocarpum. b) Parcela 2 (PS2); localidad La Pirámide (19/I/2011); UTM 19Q 331340m.E 2069149m.N; altitud, 2,395 m; temperatura del suelo al momento del muestreo, $12.8 \mathrm{C}^{\circ}$. Se encontraron dos ejemplares de $P$. occidentalis ( $<15 \mathrm{~m}$ de altura), por lo que se estableció un sólo tipo de asociación vegetal de unos $0.5 \mathrm{~m}$ de altura, con una cobertura aproximada de $85-90 \%$, dominado por $D$. domingensis y Lycopodiella cernua.

Colectas e identificación. Las arañas fueron colectadas manualmente por cinco personas (siempre las mismas y de forma simultánea) durante 21 minutos en cada cuadrícula. Se muestreó desde el dosel del pajón $(0.5-0.6 \mathrm{~m})$ hasta los $2 \mathrm{~cm}$ bajo el suelo, en horas de la mañana (08:00-10:30 hrs.). Los especímenes se preservaron en alcohol etílico al 70\% y fueron identificados hasta el nivel de especie siguiendo la literatura especializada, veinte de ellas fueron separadas como morfoespecies. Para el ordenamiento taxonómico se siguió a Platnick (2014). Los especímenes fueron depositados en la colección aracnológica del Museo Nacional de Historia Natural "Prof. Eugenio de Jesús Marcano" (MNHNSD), República Dominicana.

Abundancia y riqueza de especies. Se calculó la riqueza (S) como el número de especies encontradas en cada temporada; además, se estimó la abundancia proporcional $(\mathrm{n} / \mathrm{N})$ de acuerdo con Halffter et al. (2001). La diversidad de especies se calculó por parcela usando el índice de Shannon (Magurran, 2004). También se calcularon (por parcela) los índices de Dominancia de Simpson y de Equidad de Pielou (Halffter et al., 2001). Para estimar la similitud de las comunidades entre las temporadas se empleó el índice de Similitud de Jaccard (Halffter et al., 2001). Se realizó una prueba t de Student de dos colas, utilizando el software InfoStat versión 2014 (Di Rienzo et al., 2014), para probar la hipótesis $(\alpha=0.05)$ de que la temporada influye sobre la diversidad y la abundancia de arañas en la sabana de pajón. Para la agrupación por gremios se siguió a Uetz et al. (1999).

Se calcularon los estimadores de riqueza de especies: ACE, Chao 1 y 2, y Jackknife 1; utilizando el programa EstimateS versión 9 (Colwell, 2013). Para conocer las curvas de acumulación de especies se ajustó la función que se describe según la ecuación de Clench $\mathrm{Sn}=\mathrm{a} \cdot \mathrm{n} /$ $(1+b \cdot n)$, donde a y b son los parámetros de la función (Jiménez-Valverde y Hortal, 2003); para ello se utilizó el programa Statistica Ver. 12. La proporción de fauna registrada (q) se calculó utilizando la fórmula $\mathrm{q}=\mathrm{S}_{\mathrm{n}} /(\mathrm{a} / \mathrm{b})$, siendo el esfuerzo de muestreo $\mathrm{n}=10$.

\section{RESULTADOS}

En general, se colectaron 222 especímenes pertenecientes a 19 familias, 23 géneros y 35 especies, de las cuales ocho son endémicas de La Hispaniola (Tabla 1). Las familias con mayor riqueza de especies fueron Linyphiidae, con seis y Anyphaenidae, con cuatro. En cuanto a la abundancia, Anyphaenidae registró el 29.94\% de los especímenes y Salticidae el 17.84\%. Las especies Barronopsis barrowsi (Gerstch) y Pensacola electa Bryant fueron las más abundantes, registrando el 14.19 y $13.88 \%$, respectivamente. Se encontraron 16 especies comunes a las dos temporadas, mientras que diez fueron exclusivas de la temporada de lluvias y nueve de la seca. Las familias Cyrtaucheniidae y Theraphosidae fueron encontradas solo en la temporada seca, en tanto que Corinnidae, Hahniidae y Liocranidae solo se encontraron en la de lluvias. 
Se colectaron 116 especímenes en la temporada lluviosa, distribuidos en 17 familias y 26 especies, siendo Linyphiidae la familia que presentó el mayor número de especies (cuatro) y Salticidae la más abundante (21.54\%); las especies B. barrowsi (Gerstch) y P. electa Bryant fueron las más abundantes registrando el 18.96 y el $15.51 \%$, respectivamente (Tabla 1). Por otro lado, en la temporada seca se colectaron 106 especímenes distribuidos en 16 familias y 25 especies; en este caso Anyphaenidae fue la familia más diversa y abundante (cuatro especies y un 44.31\%) y las especies Anyphaena pusilla Bryant y Anyphaena sp. fueron las más abundantes en esta temporada (21.69 y $16.03 \%)$.

Tabla 1. Resumen de la abundancia proporcional ( $\mathrm{P}_{\mathrm{i}}$, en porcentaje), riqueza de especies $(\mathrm{S})$ e índice de Similitud de Jaccard ( $\mathrm{Ij}$ ). Valores medios (desviación estándar: DE) por parcela (dos en cada temporada), de los índices Diversidad de Shannon (H'), Dominancia de Simpson $(\lambda)$ y Equidad de Pielou $\left(J^{\prime}\right)$.

\begin{tabular}{|c|c|c|}
\hline \multirow{2}{*}{ Familia/Especie } & Temporada de Lluvias & Temporada Seca \\
\hline & $\mathrm{P}_{\mathrm{i}}(\%)$ & $\mathrm{P}_{\mathrm{i}}(\%)$ \\
\hline AGELENIDAE & \multicolumn{2}{|c|}{ Tejedora telas sábanas } \\
\hline Barronopsis barrowsi & 18.96 & 9.43 \\
\hline ANYPHAENIDAE & \multicolumn{2}{|c|}{ Cazadora corredora de follaje } \\
\hline Anyphaena dominicana $* *$ & 6.03 & 1.88 \\
\hline Anyphaena pusilla ** & 0.00 & 21.69 \\
\hline Anyphaena sp. & 7.75 & 16.03 \\
\hline Anyphaenidae sp1. & 1.72 & 4.71 \\
\hline CLUBIONIDAE & \multicolumn{2}{|c|}{ Cazadora corredora de follaje } \\
\hline Clubiona sp. & 0.00 & 1.88 \\
\hline Clubionidae sp1. & 0.86 & 0.00 \\
\hline CORINNIDAE & \multicolumn{2}{|c|}{ Cazadora corredora de suelo } \\
\hline Corinnidae sp1. & 2.58 & 0.00 \\
\hline Trachelas bicolor ** & 0.86 & 0.00 \\
\hline CYRTAUCHENIIDAE & \multicolumn{2}{|c|}{ Cazadora } \\
\hline Bolostromus sp. & 0.00 & 0.94 \\
\hline GNAPHOSIDAE & \multicolumn{2}{|c|}{ Cazadora corredora de suelo } \\
\hline Zelotes sp. & 0.86 & 0.94 \\
\hline HAHNIIDAE & \multicolumn{2}{|c|}{ Tejedora telas sábanas } \\
\hline Hahniidae sp1. & 0.86 & 0.00 \\
\hline LINYPHIIDAE & \multicolumn{2}{|c|}{ Tejedora errante telas sábanas } \\
\hline Ceraticelus paludigenus & 1.72 & 2.83 \\
\hline Ceraticelus sp. & 0.00 & 0.94 \\
\hline Grammonota $\mathrm{sp}$. & 0.00 & 0.94 \\
\hline Microneta sp. & 0.86 & 0.00 \\
\hline Tapinopa bilineata & 1.72 & 0.00 \\
\hline Tapinopa sp. & 1.72 & 0.00 \\
\hline
\end{tabular}


Tabla 1 (continuación).

\begin{tabular}{|c|c|c|}
\hline \multirow{2}{*}{ Familia/Especie } & Temporada de Lluvias & Temporada Seca \\
\hline & $\mathrm{P}_{\mathrm{i}}(\%)$ & $\mathrm{P}_{\mathrm{i}}(\%)$ \\
\hline LIOCRANIDAE & \multicolumn{2}{|c|}{ Cazadora } \\
\hline Lausus pulchellus ** & 0.86 & 0.00 \\
\hline LYCOSIDAE & \multicolumn{2}{|c|}{ Cazadora corredora de suelo } \\
\hline Pardosa portoricensis & 8.62 & 5.66 \\
\hline MITURGIDAE & \multicolumn{2}{|c|}{ Cazadora corredora de suelo } \\
\hline Miturgidae sp1. & 1.72 & 0.94 \\
\hline Teminius monticola $* *$ & 0.00 & 0.94 \\
\hline OCHYROCERATIDAE & \multicolumn{2}{|c|}{ Tejedora } \\
\hline Ochyrocera cachote ** & 2.58 & 2.83 \\
\hline OONOPIDAE & \multicolumn{2}{|c|}{ Cazadora } \\
\hline Heteroonops sp. & 0.86 & 0.94 \\
\hline PHOLCIDAE & \multicolumn{2}{|c|}{ Tejedora telas irregulares } \\
\hline Modisimus sp. & 8.62 & 2.83 \\
\hline SALTICIDAE & \multicolumn{2}{|c|}{ Cazadora al asecho } \\
\hline Pensacola electa ** & 15.51 & 12.26 \\
\hline Siloca electa $* *$ & 6.03 & 1.88 \\
\hline TETRAGNATHIDAE & \multicolumn{2}{|c|}{ Tejedora telas orbiculares } \\
\hline Tetragnatha tenuissima & 0.86 & 1.88 \\
\hline Tetragnatha sp. & 0.86 & 3.77 \\
\hline Tetragnathidae sp1. & 2.58 & 0.00 \\
\hline THERAPHOSIDAE & \multicolumn{2}{|c|}{ Cazadora } \\
\hline Phormictopus sp. & 0.00 & 0.94 \\
\hline Theraphosidae sp1. & 0.00 & 0.94 \\
\hline THERIDIIDAE & \multicolumn{2}{|c|}{ Tejedora telas irregulares } \\
\hline Faiditus sp. & 0.86 & 0.00 \\
\hline Steatoda grossa & 0.00 & 0.94 \\
\hline THOMISIDAE & \multicolumn{2}{|c|}{ Cazadora por emboscada } \\
\hline Xysticus pellax & 3.44 & 0.94 \\
\hline Total de especímenes (N) & 116 & 106 \\
\hline Riqueza de especies (S) & 26 & 25 \\
\hline $\mathrm{H}^{\prime}$ & $2.42(\mathrm{DE}= \pm 0.24)$ & $2.41(\mathrm{DE}= \pm 0.10)$ \\
\hline$\lambda$ & $0.12(\mathrm{DE}= \pm 0.03)$ & $0.12(\mathrm{DE}= \pm 0.02)$ \\
\hline $\mathrm{J}^{\prime}$ & $0.85(\mathrm{DE}= \pm 0.04)$ & $0.86(\mathrm{DE}= \pm 0.04)$ \\
\hline $\mathrm{Ij}$ & \multicolumn{2}{|c|}{0.46} \\
\hline
\end{tabular}

A la derecha de cada familia se incluye el gremio de acuerdo con Uetz et al. (1999). Las familias y especies dentro de éstas figuran ordenadas alfabéticamente. $* *=$ endémica de La Hispaniola. 


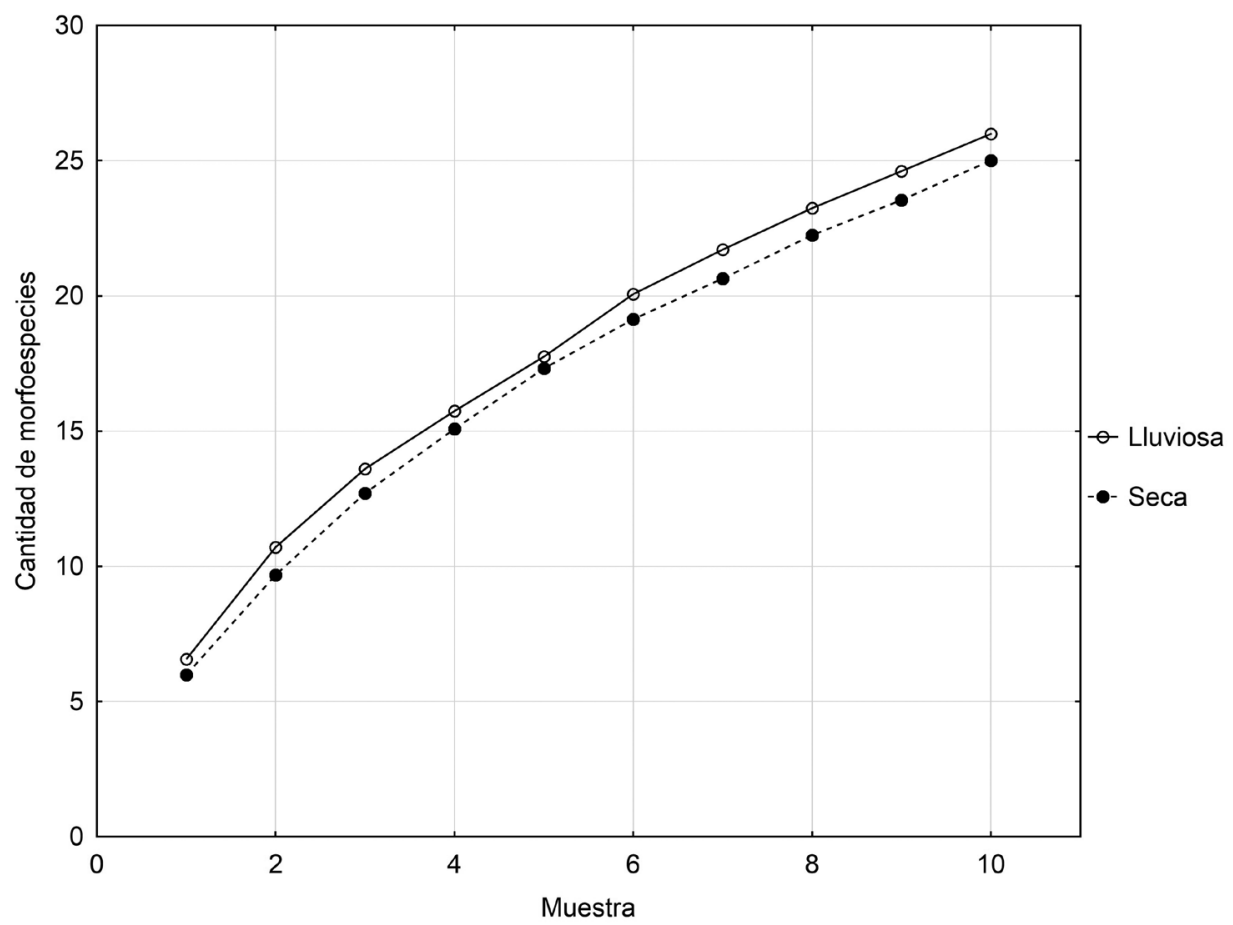

Figura 1. Curvas de acumulación de especies ajustadas a la ecuación de Clench. Temporada lluviosa: Sobs $=26, R^{2}=0.994, a=6.68$, $\mathrm{b}=0.16$, pendiente $=0.99$. Temporada seca: $\mathrm{Sobs}=25, \mathrm{R}^{2}=0.997, \mathrm{a}=6.13, \mathrm{~b}=0.15$, pendiente $=0.98$.

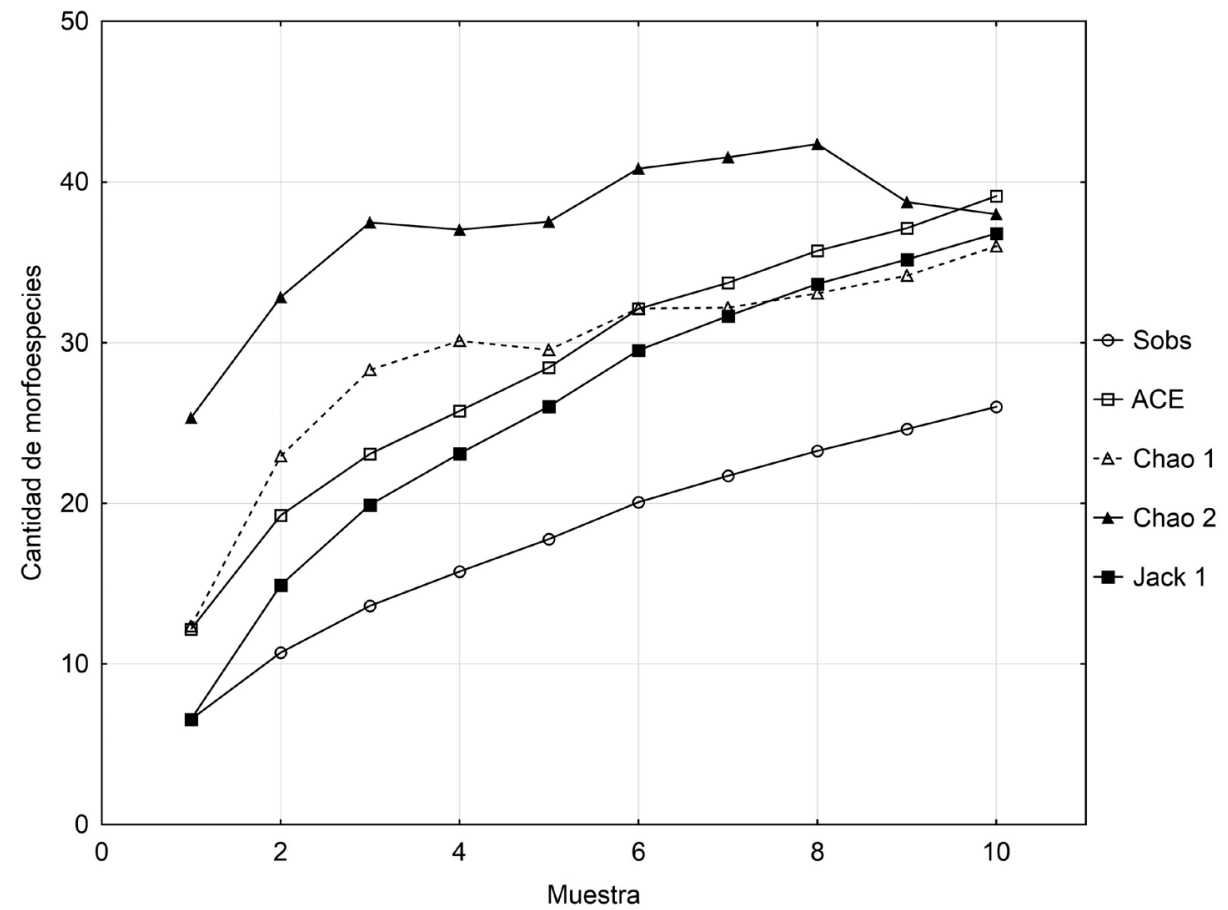

Figura 2. Curvas de estimación de especies para la temporada lluviosa luego de 100 aleatorizaciones. 


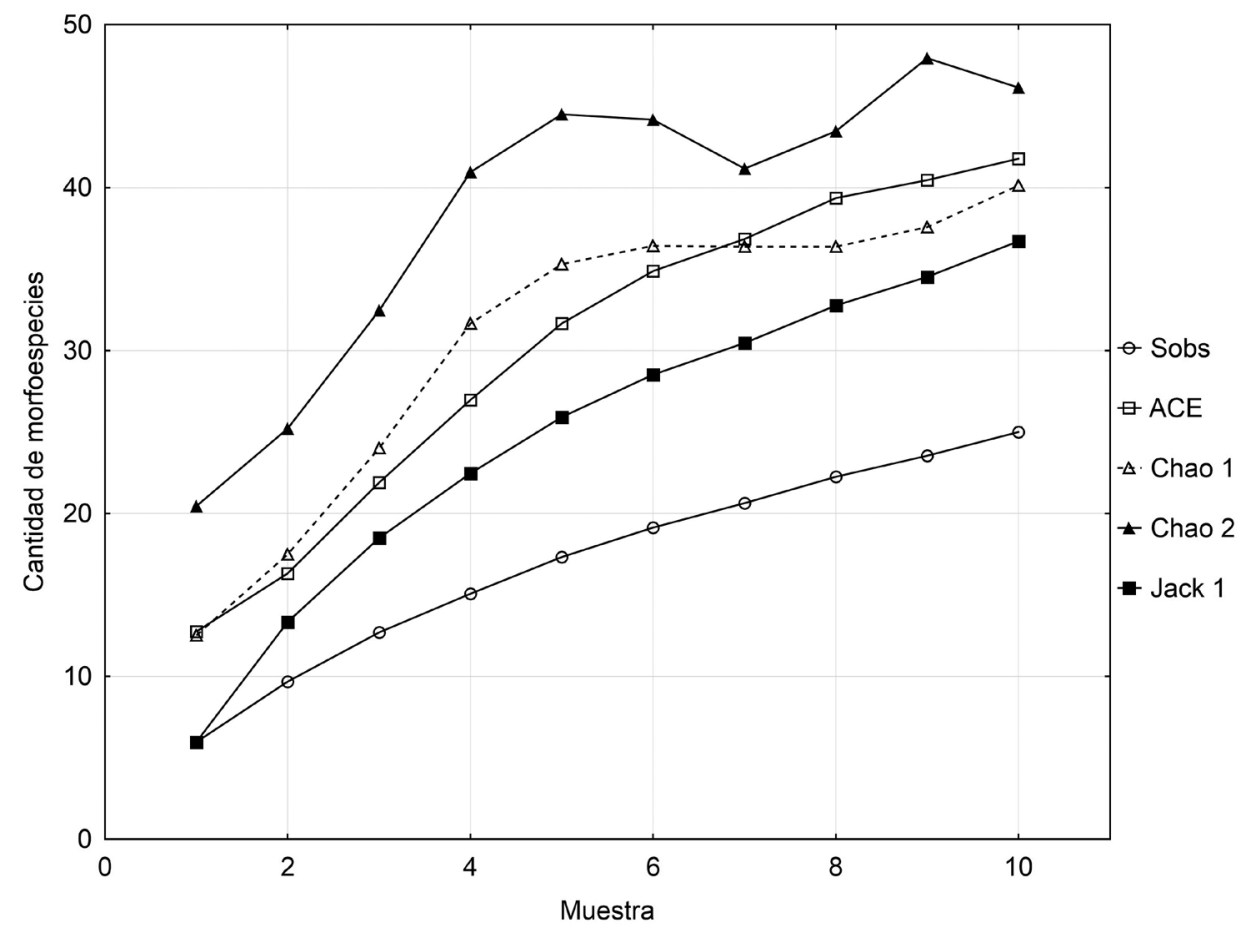

Figura 3. Curvas de estimación de especies para la temporada seca luego de 100 aleatorizaciones.

Atendiendo a los gremios, de manera general, se registró una mayor diversidad y abundancia de arañas cazadoras (20 especies y un $65.6 \%$, respectivamente) en relación a las tejedoras (15; $34.4 \%$ ). La diversidad de las arañas cazadoras fue mayor en ambas temporadas con 14/12 (cazadoras/tejedoras) en la lluviosa y 16/14 en la seca, algo observado también en la abundancia: $57.7 \%-42.3 \%$ (cazadoras-tejedoras) en la lluviosa y $73.5 \%-26.5 \%$ en la seca.

No se encontraron diferencias significativas en los índices Diversidad de Shannon, Dominancia de Simpson y Equidad de Pielou entre las temporadas (Tabla 1). El mayor y menor valor del índice de Shannon se registró en la temporada de lluvias (2.65 y 2.19, respectivamente); la prueba t de Student mostró que no hubo diferencias significativas para este índice entre las temporadas $(\mathrm{T}=0.18 ; P=0.86)$. El índice de Simpson también registró su mayor y menor valor en la temporada de lluvias ( 0.15 y 0.09 , respectivamente), la prueba $t$ de Student mostró que tampoco hubo diferencias significativas entre las temporadas $(\mathrm{T}=0.00 ; P>0.99)$. En el caso del índice de Pielou, este registró su mayor valor en la temporada seca (0.90) y el menor en la de lluvias (0.81), al igual que en los casos anteriores, la prueba t de Student indicó que no hubo diferencias significativas entre las temporadas $(\mathrm{T}=-0.85 ; P=0.41)$. El valor del índice de Similitud de Jaccard entre las temporadas fue de 0.46 .

Las pendientes obtenidas en las curvas de acumulación ajustadas para cada temporada resultaron muy por encima de 0.1 (0.99 en la temporada de lluvias y 0.98 en la seca; ver Fig. 1). La proporción de fauna registrada para la temporada lluviosa fue del $62 \%$, mientras que para la seca fue del 61\%. La mayor cantidad de especies fue estimada por Chao 2 (46 especies para la temporada seca), mientras que la menor cantidad fue de 36, coincidiendo Chao 1 y Jackknife 1 (temporada lluviosa) y Chao 1 (temporada seca); ver Tabla 2 y Figs. 2 y 3. 
Tabla 2. Valores de los estimadores de riqueza de especies calculados para cada temporada, así como la proporción de fauna registrada (q), basados en los datos de las 10 cuadrículas de 2x2 m muestreadas en cada una. El valor de los estimadores representa la media de 100 aleatorizaciones.

\begin{tabular}{|l|c|c|}
\cline { 2 - 3 } \multicolumn{1}{c|}{} & Temporada de lluvias & Temporada seca \\
\hline Sobs & 26 & 25 \\
\hline Total de especímenes (N) & 116 & 106 \\
\hline \% Singletons & 38.5 & 44 \\
\hline \% Uniques & 46.1 & 52 \\
\hline ACE & 39.11 & 41.77 \\
\hline Chao 1 & 36 & 40.12 \\
\hline Chao 2 & 38 & 46.12 \\
\hline Jackknife 1 & 36.80 & 36.70 \\
\hline $\mathrm{q}$ & $62 \%$ & $61 \%$ \\
\hline
\end{tabular}

\section{DISCUSIÓN}

Los resultados del presente estudio indican que hubo una distribución homogénea de los individuos en cada temporada: $H^{\prime}$ ( $\mathrm{TLL}=2.42$, TS $\left.=2.41\right) ; \lambda(0.12$ en ambas); J' (TLL $=0.85$, $\mathrm{TS}=0.86)$. La comparación entre los índices obtenidos en las temporadas no arrojó diferencias significativas $\left(\mathrm{H}^{\prime}, P=0.86 ; \lambda, P>0.99 ; \mathrm{J}^{\prime}, P=0.41\right)$. La composición específica, en cambio, sí presentó diferencias entre las temporadas (Tabla 1 ).

Muchos de los trabajos sobre estacionalidad y riqueza de arañas en ecosistemas de sabanas y pastizales no concuerdan en sus resultados debido a que en ellos se estudian ensamblajes de especies diferentes con comportamientos, presiones y amenazas diferentes. Esto se refleja en las disimilitudes entre los resultados del presente trabajo y los obtenidos por Arango et al. (2000), Sudhikumar et al. (2005) y Mineo et al. (2010), quienes encontraron que la estacionalidad influyó tanto en la riqueza como en la abundancia de arañas, observando además, que las condiciones climáticas anteriores al momento de muestreo tuvieron mayor impacto sobre las poblaciones que las encontradas durante el mismo.

Los resultados de las curvas de acumulación de especies indican que el diseño del muestreo no fue suficiente para registrar todas las especies que se esperan encontrar (Jiménez-Valverde y Hortal, 2003), sin embargo, la proporción de fauna registrada (más del 60\% en cada temporada) fue relativamente alta si se considera el reducido tamaño de la muestra. Esto último también puede observarse al comparar la cantidad de especies encontradas frente a las estimadas en cada temporada, números que no difieren tanto si se toman en cuenta las especies raras; influyendo en estas diferencias la alta proporción de singletons $(>37 \%)$ y uniques $(>45 \%)$. Partiendo de ésta alta proporción y de que solo restaron por encontrarse entre 10 y 15 especies, la diversidad real de estas sabanas no debe distar demasiado de la encontrada.

La mayor diversidad y abundancia de arañas cazadoras respecto a las tejedoras coincide con los resultados de Whitmore et al. (2002), surgiendo como una posible explicación el hecho de que las sabanas del presente estudio no ofrecen las condiciones de altura y ramificación que necesitan las arañas tejedoras (Duffey, 1962; Turnbull, 1973 En Sudhikumar et al., 2005). No obstante, dentro de las tejedoras orbiculares, la familia Tetragnathidae parece haber encontrado mecanismos que le permiten dominar en este ecosistema, ya que no se registró ningún miembro de otras familias de este gremio (Araneidae y Uloboridae), aun pese a que los autores han colectado miembros de éstas en los bosques de pinos que delimitan las sabanas de pajón. 
La alta abundancia de las familias Anyphaenidae y Salticidae (ambas cazadoras) difiere de otros resultados donde Lycosidae y Zodariidae fueron las más abundantes o estuvieron entre las tres primeras (Churchill, 1998; Sudhikumar et al., 2005; Mineo et al., 2010); aunque en el caso de Salticidae, Russell-Smith (2002) encontró una abundancia similar, ocupando ésta el primer lugar en su estudio.

Considerando que tanto la planta dominante (Danthonia domingensis) como las especies encontradas de estas familias son endémicas (i.e. Anyphaena dominicana, A. pusilla, Pensacola electa y Siloca electa), la alta abundancia de estas familias puede ser una característica de este ecosistema en donde la estructura vegetal del mismo presenta mejores oportunidades para las arañas cazadoras, en particular para estos dos gremios. Esto también se refleja en que dentro de las tejedoras, la familia Agelenidae -tejedoras de telas en sábanas- fue la más abundante y sus telas generalmente fueron observadas entre $15-20 \mathrm{~cm}$ de altura.

Se espera que estas consideraciones estimulen futuros estudios sobre la araneofauna en las sabanas de pajón, sugiriéndose muestrear los bordes de los bosques de pinos que delimitan las mismas.

\section{AGRADECIMIENTOS}

Se agradece al Fondo Nacional de Innovación y Desarrollo Científico y Tecnológico (FONDOCyT) del Ministerio de Educación Superior, Ciencia y Tecnología (MESCyT), de la República Dominicana, por el financiamiento que hizo posible este trabajo. A los investigadores del MNHNSD por la asistencia en campo. Al Dr. Giraldo Alayón, del Museo Nacional de Historia Natural, La Habana, Cuba, por su valiosa ayuda en la identificación de las arañas. A Carlos Suriel (MNHNSD), por la ayuda en la revisión del manuscrito. Alexander Sánchez Ruiz, del Centro Oriental de Ecosistemas y Biodiversidad, Cuba (BIOECO) e Instituto Butantan, Brasil, hizo importantes correcciones y sugerencias que enriquecieron el manuscrito.

\section{LITERATURA CITADA}

Alayón, G. 2011. Apuntes sobre la familia Araneidae, con la descripción de dos especies nuevas de Wagneriana (Araneae: Araneidae) de Cuba y República Dominicana. Solenodon 9: 20-28.

Arango, A. M., V. Rico-Gray y V. Parra-Tabla. 2000. Population structure, seasonality, and habitat use by the Green Lynx Spider Peucetia viridans (Oxyopidae) inhabiting Cnidoscolus aconitifolius (Euphorbiaceae). Journal of Arachnology 28: 185-194.

Bryant, E. B. 1943. The salticid spiders of Hispaniola. Bulletin of the Museum of Comparative Zoology Harvard 92: 445-529.

Bryant, E. B. 1945. The Argiopidae of Hispaniola. Bulletin of the Museum of Comparative Zoology Harvard 95: 357-422.

Bryant, E. B. 1948. The spiders of Hispaniola. Bulletin of the Museum of Comparative Zoology Harvard 100: 331-459.

Carrero Jiménez, S. A. 2014. Araneofauna de la sabana de pajón del Parque Nacional Valle Nuevo y su respuesta a los efectos del fuego y la agricultura y su relación con las temporadas lluviosa o seca del año. Tesis de Licenciatura en Biología, Universidad Autónoma de Santo Domingo, Santo Domingo, República Dominicana. 84 pp. [Sin publicar]. 
Ceballos, M. 2006. Ficha técnica del Parque Nacional Juan Bautista Pérez Rancier (Valle Nuevo). Secretaria de Estado de Medio Ambiente y Recursos Naturales, Subsecretaria de Áreas Protegidas y Biodiversidad, Dirección de Áreas Protegidas. http://www. gestiopolis.com/administracion-estrategia/ficha-tecnica-de-un-parque-natural.htm. Accedido: 03-marzo-2014.

Coddington, J. A. y H. W. Levi. 1991. Systematics and evolution of spiders (Araneae). Annual Review of Ecology and Systematics 22: 565-92.

Colwell, R. K. 2013. EstimateS: Statistical estimation of species richness and shared species from samples. Version 9. User's Guide and application published at: http://purl.oclc.org/ estimates.

Churchill, T. B. 1998. Spiders as ecological indicators in the Australian tropics: family distribution patterns along rainfall and grazing gradients. Proceedings of the 17th European Colloquium of Arachnology, Edinburgh 1997.

Deza, M. y J. M. Andía. 2009. Diversidad y riqueza de especies de la familia Araneidae (Arachnida, Araneae) en Cicra (Madre de Dios-Perú). Ecología Aplicada 8 (2): 81-91.

Di Rienzo, J. A., F. Casanoves, M. G. Balzarini, L. González, M. Tablada, C. W. Robledo. InfoStat versión 2014. Grupo InfoStat, FCA, Universidad Nacional de Córdoba, Argentina. URL http://www.infostat.com.ar.

Duffey, E. 1962. A population study of spiders in limestone grass land: The field layer fauna. Oikos 13:15-34.

Halffter, G., C. E. Moreno y E. O. Pineda. 2001. Manual para evaluación de la biodiversidad en Reservas de la Biosfera. M\&T-Manuales y Tesis SEA, vol. 2. Zaragoza, 80 pp.

Jiménez-Valverde, A. y J. Hortal. 2003. Las curvas de acumulación de especies y la necesidad de evaluar la calidad de los inventarios biológicos. Revista Ibérica de Aracnología 8: 151-161.

Lubin, Y. D. 1978. Seasonal abundance and diversity of wed-building spiders in relation to habitat structure on Barro Colorado Island, Panama. Journal of Arachnology 6: 31-51.

Magurran, A. E. 2004. Measuring biological diversity. Blackwell Science Ltd., 256 pp.

Mineo, M. F., K. Del-Claro y A. D. Brescovit. 2010. Seasonal variation of ground spiders in a Brasil Savanna. Zoologia 27 (3): 353-362.

Modiba, M. A., S. M. Dippenaar y A. S. Dippenaar-Schoeman. 2005. A checklist of spiders from Sovenga Hill, an inselberg in the Savanna Biome, Limpopo Province, South Africa (Arachnida: Araneae). Koedoe 48: 109-115.

Núñez, F., N. Ramírez, M. McPherson y F. Portorreal. 2006. Plan de conservación del Parque Nacional Juan Bautista Pérez Rancier (Valle Nuevo). Editora Amigo del Hogar. Santo Domingo, República Dominicana. 87 pp.

Peguero, B. 2010. Composición y estructura de la vegetación en ocho parcelas de sabana de Pajón, Valle Nuevo. Informe vegetación FONDOCyT -Valle Nuevo. Proyecto FONDOCyT 2009 "Ecología de la fauna asociada al suelo en la sabana de pajón del Parque Nacional 
Juan Bautista Pérez Rancier (Valle Nuevo) y su respuesta a los impactos de la agricultura y el fuego". $49 \mathrm{pp}$.

Platnick, N. I. y M. U. Shadab. 1974. A revision of the bispinosus and bicolor groups of the spider genus Trachelas (Araneae, Clubionidae) in North and Central America and the West Indies. American Museum Novitates 2560: 1-34.

Platnick, N. I. 2014. The world spider catalog, version 14.5. American Museum of Natural History, online at http://research.amnh.org/entomology/spiders/catalog/index.html DOI: 10.5531/db.iz.0001. Accedido: 23-I-2014.

Russell-Smith, A. 2002. A comparison of the diversity and composition of ground-active spiders in Mkomazi Game Reserve, Tanzania and Etosha National Park, Namibia. Journal of Arachnology 30: 383-388.

Sánchez-Ruiz, A., C. Suriel y G. de los Santos. 2009. Muestreo postfuego de artrópodos de suelo en bosques de pinos del Parque Nacional José del Carmen Ramírez, República Dominicana. Novitates Caribaea 2: 30-39.

Sudhikumar, A. V., M. J. Mathew, E. Sunish y P. A. Sebastian. 2005. Seasonal variation in spider abundance in Kuttanad rice agroecosystem, Kerala, India (Araneae). Acta Zoologica Bulgarica 1: 181-190.

Turnbull, A. L. 1973. Ecology of the true spiders (Araneomorphae). Annual Review of Entomology 18: 305-348.

Uetz, G. W., J. Halaj y A. B. Cady. 1999. Guild structure of spiders in major crops. Journal of Arachnology 27: 270-280.

Whitmore, C., R. Slotow, T. E. Crouch y A. S. Dippenaar-Schoeman. 2002. Diversity of spiders (Araneae) in a savanna reserve, Northern Province, South Africa. Journal of Arachnology 30: 344-356.

[Recibido: 20 de febrero, 2014. Aceptado para publicación: 11 de septiembre, 2014] 